\title{
Validation of fine needle aspiration smear processing method for onsite immediate assessment
}

\author{
C. S. P. Sosai ${ }^{1}$, A. A. D. C. Perera ${ }^{1}$, A. A. S. Priyangika ${ }^{1}$, K. K. T. H. Perera ${ }^{1}$, E. H. Siriweera ${ }^{2}$ \\ S. Wijethunga ${ }^{2}$ and A. A. H. Priyani ${ }^{3}$ \\ Department of Pathology, District General Hospital, Gampaha, Sri Lanka ${ }^{1}$ \\ Department of Pathology, Faculty of medicine, University of Peradeniya, Sri Lanka ${ }^{2}$ \\ Department of Pathology, Faculty of Medicine, University of Colombo, Sri Lanka ${ }^{3}$ \\ DOI: http://doi.org/10.4038/jdp.v11i2.7715
}

\section{Introduction}

A suitable protocol to ensure rapid processing of smears for on-site immediate assessment (OSIA) is not available in Sri Lanka. The objective of this study was to validate the processing method of fine needle aspirates (FNA) for OSIA.

\section{Methodology}

Thirty five FNA obtained were processed as follows. The smear prepared from the first needle pass was fixed in 95\% ethyl alcohol and stained with freshly prepared Harris haematoxylin for one minute each and assessed for adequacy of cellularity. It was then fixed for 20 minutes in the same fixative and stained by the Papanicolaou staining protocol (PSP). The smear prepared from the second needle pass was routinely processed and stained by PSP. Both smears were assessed by three histopathologists ( $A, B$ and $C$ ) independently and blind to the processing method for quality indicators, including air drying (ADpresence/absence), nuclear differentiation(NDclarity of visibility of chromatin pattern as 1poor, 2-intermediate and 3-clear,) and cytoplasmic clarity (CC-cell outline and clarity as 1-poor, 2- intermediate and 3-clear). Final quality of smear for reporting was categorized as satisfactory for reporting (absent $A D$ and ND 2-3, CC 2-3), unsatisfactory but can report (absent $A D$ and ND 1-2, CC 1-2) and unsatisfactory, cannot report (presence of $A D$ and ND1, CC1). Statistical analysis was done using chi-square test.

\section{Results}

Of the 35 OSIA smears, pathologist A, B and C found $(35 / 35) 100 \%$, (26/35) $74.29 \%$, and $(28 / 35) 80 \%$ to be satisfactory for reporting, and $(0 / 35) 0 \%,(1 / 35) 2.86 \%$, and $(1 / 35) 2.86 \%$ unsatisfactory for reporting. Of the 35 routinely processed smears, pathologist $A, B$ and $C$ found $(29 / 35) \quad 82.9 \%, \quad(24 / 35) \quad 68.6 \%$ and $(16 / 35)$ $45.7 \%$ to be satisfactory for reporting: (4/35)11.4\%, (4/35)11.4\% and (3/35)8.6\% to be unsatisfactory for reporting.

\section{Conclusion}

No significant difference $(p<0.05)$ was found by the three pathologists in the quality parameters of the smears from the two processing methods thus, validating the one minute processing method as comparable to the routine processing method. 